\title{
Low Temperature Surface-Mediated Growth of Graphene On Ultra-Thin Metal Catalysts
}

\author{
Sam Olson ${ }^{1}$, Otto Zietz ${ }^{1}$, Brendan Coyne ${ }^{1}$ and Jun Jiao ${ }^{1}$ \\ 1. Department of Mechanical and Materials Engineering, Portland State University, Portland, USA.
}

Graphene, a single layer of $\mathrm{sp}^{2}$-bonded carbon atoms arranged in a hexagonal lattice, has a myriad of potential applications in the semiconductor industry. It has excellent electron mobility, allowing for the creation of graphene-based field-effect transistors (FETs), and is also capable of acting as a highly effective diffusion barrier [1,2]. In order to enable use of these properties in modern microelectronic fabrication processes, a low-temperature graphene synthesis process that is capable of directly forming graphene onto targeted materials is needed. Current chemical vapor deposition (CVD) processes use thick (200-2,000 nm) catalysts and require growth temperatures exceeding $700{ }^{\circ} \mathrm{C}$ to produce graphene. Here, a method for CVD-fabricating graphene films at a temperature of $450{ }^{\circ} \mathrm{C}$ is presented using a 1 $\mathrm{nm} \mathrm{Ni} / \mathrm{Au}$ catalyst, presenting an avenue toward low-temperature direct growth.

To achieve this growth, $1 \mathrm{~nm}$ of a $\mathrm{Ni} / \mathrm{Au}$ alloy was deposited onto a $\mathrm{Si} / \mathrm{SiO}_{2}$ wafer via electron beam evaporation. The wafer was then heated to $450{ }^{\circ} \mathrm{C}$ in a CVD system under $\mathrm{H}_{2}$. Upon reaching $450{ }^{\circ} \mathrm{C}$, $\mathrm{H}_{2}$ flow was halted and $0.1 \mathrm{sccm}$ of the carbon precursor $\left(\mathrm{C}_{2} \mathrm{H}_{2}\right)$ was introduced for varying durations. SEM inspection of the wafer post-growth showed that the metal catalyst conglomerated into circular islands (Fig. 1). Raman spectroscopy of the post-growth wafers showed a consistent 2D peak full-width half-maximum $\left(\mathrm{FWHM}_{2 \mathrm{D}}\right)$ with extended growth durations, wherein a thinner $\mathrm{FWHM}_{2 \mathrm{D}}$ indicates fewer graphene layers [3]. Comparatively, SEM micrographs and Raman spectra of growths conducted on a 50 nm substrate for 0.5-3 minutes are shown in Fig. 2. The $\mathrm{FWHM}_{2 \mathrm{D}}$ of these spectra become significantly broader with longer growth times, indicating increasing layer number. The SEM micrographs of the 50 nm catalysts show grains (Fig. 2) in lieu of the catalyst islands observed on the $1 \mathrm{~nm}$ samples (Fig. 1).

Raman spectra representative of growths conducted on a $1 \mathrm{~nm}$ catalyst and a more typical, thicker catalyst are compared in Fig. 3 (a). The spectrum of the $1 \mathrm{~nm}$ sample displays drastic peak shifting, which occurs when the crystal structure of the graphene experiences axial stress [4]. The Raman spectrum of the $1 \mathrm{~nm}$ catalyst also displays a splitting of the G peak, a feature found on graphene with broken planar symmetry [5]. The observation of both of these Raman features indicates that the graphene on the $1 \mathrm{~nm}$ catalyst is stressed and lacks planar symmetry. A proposed growth mechanism is elucidated in Fig 3 (b). Upon introduction of the carbon precursor, the ultra-thin catalyst immediately grows layers of graphene without precipitation of further layers over time due to the absence of a bulk volume. The catalyst under the graphene then aggregates into islands, inducing stress on the graphene sheets and breaking their planar symmetry as the sheets sag in the gaps between the catalyst islands.

The consistency of the Raman spectra with extended growth time on the $1 \mathrm{~nm}$ catalyst indicates that the growth mechanism is surface-mediated, as there is not an appreciable bulk volume of catalyst for large amounts of carbon to dissolve into and subsequently precipitate out of. In comparison, the $50 \mathrm{~nm}$ catalyst presents a bulk volume capable of dissolving more carbon. This increased amount of dissolved carbon leads to additional layers of graphene precipitating out of the catalyst. These results indicate that $450{ }^{\circ} \mathrm{C}$ growths on ultra-thin catalysts present an avenue toward direct growth of reduced layer-number graphene due to the absence of an appreciable carbon reservoir [6]. 
References:

[1] H. Hideki et al, Journal of Applied Physics 116 (2014), p. 083703.

[2] B. Nguyen et al, Applied Physics Letters 104 (2014), p. 082105.

[3] A.C. Ferrari, Solid State Communications 143 (2007), p. 47.

[4] J. Zabel et al, Nano Letters 12 (2012), p. 617.

[5] M. Dresselhaus et al, Nano Letters 10 (2010), p. 751.

[6] This study is supported in part by Oregon Metals Initiative and NSF awards No. 1711994 and No. 1560383. The authors would also like to thank Intel Corporation for their support.

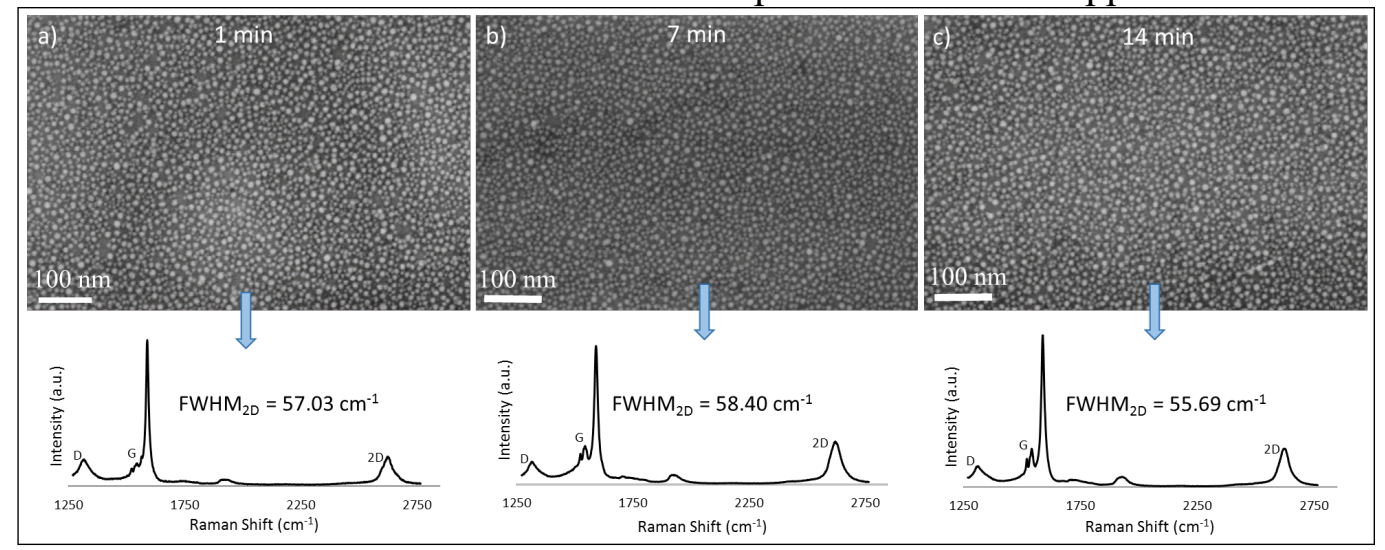

Figure 1: Comparison of SEM/Raman of growths conducted for (a) 1 minute, (b) 7 minutes, and (c) 14 minutes on $1 \mathrm{~nm}$ catalyst. Note the consistency of both the micrographs and the Raman spectra.
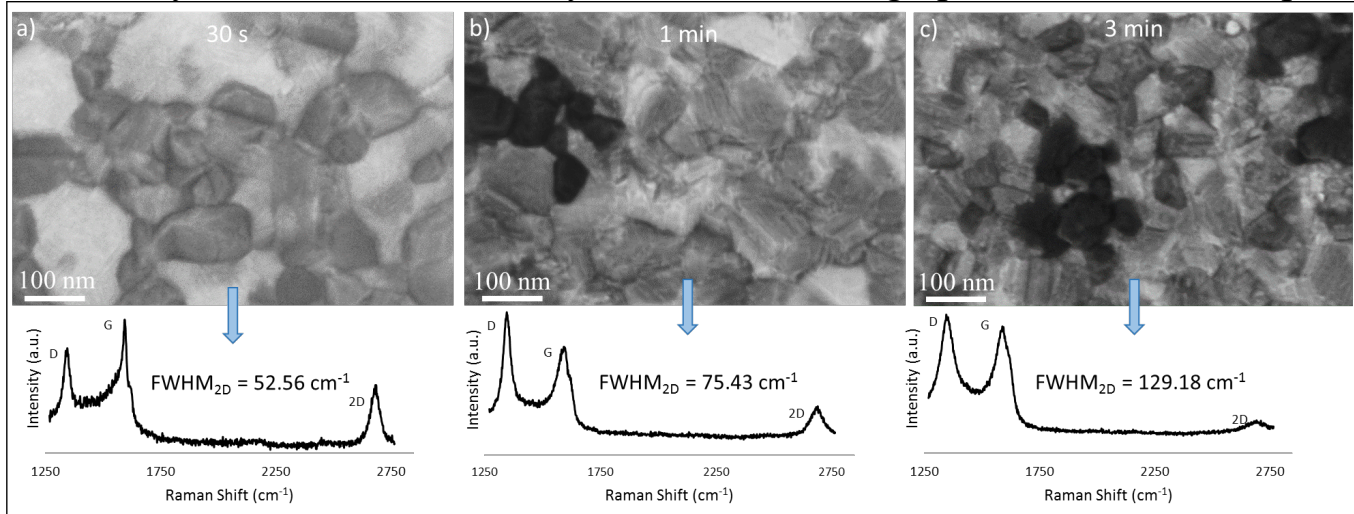

Figure 2: Comparison of SEM/Raman of growths conducted for (a) 30 seconds, (b) 1 minute, and (c) 3 minutes on $50 \mathrm{~nm}$ catalyst. Note the widening of the 2D peak in the Raman spectra and the absence of catalyst islands in the SEM. Darker areas in the SEM micrographs are areas with more graphene layers

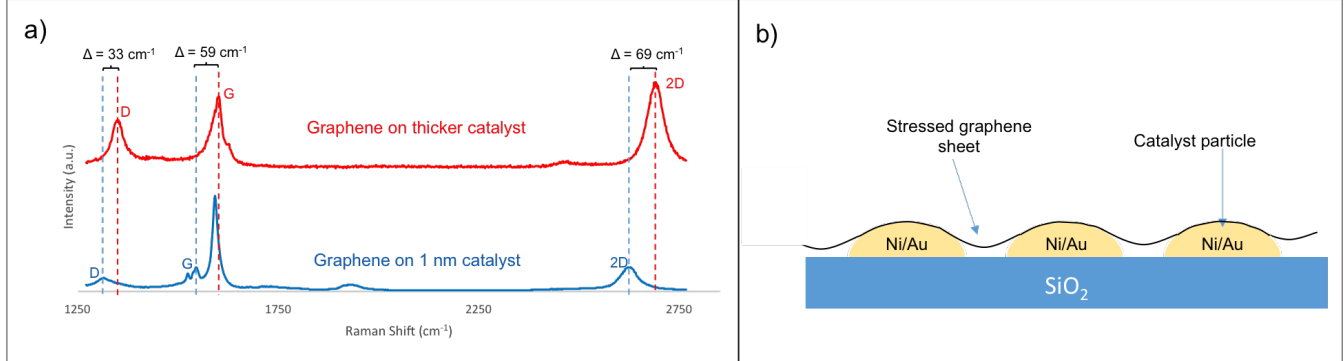

Figure 3: (a) Raman of graphene on $1 \mathrm{~nm}$ catalyst (blue) compared to Raman of graphene grown on a thicker catalyst (red). Note the peak shifting and $\mathrm{G}$ peak splitting that occurs on the $1 \mathrm{~nm}$ catalyst. (b) Proposed mechanism of graphene growth on ultra-thin catalyst. 